\title{
Bioinformatics Analysis of the Phytoene Dehydrogenase Gene in Potato (Solanum tuberosum)
}

\author{
Ping Zhou ${ }^{1,2}$, Jie Ma ${ }^{1,2}$, Rui Wu ${ }^{2}$, Min Jiang ${ }^{1}$, Meiling Wu ${ }^{2}$, Chunyan Chen ${ }^{2}$, Chaohai Wang ${ }^{2}$, \\ and Bo Sun ${ }^{1, *}$ \\ ${ }^{1}$ College of Horticulture, Sichuan Agricultural University, Chengdu 611130, China; \\ ${ }^{2}$ Bijie Institute of Agricultural Science, Bijie 551700, China. \\ *Correspondence author, E-mail addresses: htang@sicau.edu.cn.
}

Keywords: Potato, carotenoids, phytoene dehydrogenase (PDS), bioinformatics analysis.

\begin{abstract}
Phytoene Dehydrogenase (PDS) is an important enzyme in carotenoid biosynthesis. Here, the Solanum tuberosum PDS (StPDS) gene sequences were obtained from Spud DB database, and preformed for bioinformatics analysis. The StPDS gene mapped to chromosomes 3, and contains an open reading frame of 1,752 bp that encodes a 583-amino acid protein with a calculated molecular mass of $64.97 \mathrm{kD}$ and an isoelectric point (pI) of 6.41. Subcellular localization predicted the StPDS gene was in the chloroplast. The conserved domain of the StPDS protein is PLN02612. The PDS protein is most closely related to Solanum pennellii. The findings of the present study provide a molecular basis for the elucidation of PDS gene function in potato.
\end{abstract}

\section{Introduction}

Potato (Solanum tuberosum) is ranked as the third most important food crop in the world. Potato is not only of importance as a food crop, and also one of the major crops grown for starch production [1]. It yields a high-energy output per hectare, and is a rich source of nutrients, including carbohydrates, and carotenoids [1-2].

Carotenoids, which are synthesized in various photosynthetic and non-photosynthetic organisms, including algae, plants, and some bacteria and fungi, are a class of 40-carbon hydrocarbon compounds derived from a terpenoid precursor [3-5]. Carotenoids represent a diverse group of pigments that contribute to the red, orange, and yellow colors of various horticultural plants, contributing to their economic value [5-6]. Carotenoids participate in various plant physiological processes, including growth, development, and responses to multiple environmental factors. In green tissues, these act as accessory pigments for the assembly of photosystems and light-harvesting antenna complexes and also have photoprotective functions during photosynthesis [3,5]. In non-green tissues, these confer distinct colors to specialized plastids known as chromoplasts, which contribute to the sundry colors in plants $[4,6]$. In addition, carotenoids are the precursors of numerous volatile flavor compounds and phytohormones such as abscisic acid (ABA) and strigolactones [4]. Carotenoids are also essential compounds of human diets, primarily as a precursor of vitamin A [7]. Moreover, carotenoid intake could also reduce the risk for cancer and cardiovascular disease [8-9].

Phytoene Dehydrogenase (PDS) is one of the essential enzymes in carotenoid biosynthesis, catalyzing the colorless phytoene into the $\zeta$-carotene [5]. The genes encoding the PDS protein have been isolated in various plant species, including Arabidopsis, tomato, citrus, strawberry, and Strelitzia reginae [10-11]. To date, research studies on PDS in potato are limited. In the present study, the PDS gene sequence of potato was obtained from web database, and then bioinformatics analysis of the PDS gene was analyzed. The present study aimed to establish the foundation for further studies on the molecular mechanism of PDS in potato. 


\section{Materials and methods}

\subsection{Sequence Obtained of the StPDS Gene.}

The genomic DNA and mRNA sequences of PDS gene of potato were downloaded and obtained from The Spud DB database (http://solanaceae.plantbiology.msu.edu), and then used to subsequent bioinformatic analysis.

\subsection{Bioinformatics Analysis of the StPDS Gene.}

The amino acid sequence, protein molecular weight, isoelectric point, stability index, and hydrophobicity of the StPDS gene were analyzed and predicted by ExPASy (http://web.expasy.org) and NCBI (https://www.ncbi.nlm.nih.gov/). Subcellular localization was predicted by WoLF PSORT (http://www.genscript.com/wolf-psort.html). The conserved domain were predicted by NCBI (https://www.ncbi.nlm.nih.gov/Structure/cdd/wrpsb.cgi). Phylogenetic tree analysis of the PDS proteins was executed in MEGA 6.0 using the neighbor-joining (NJ) method.

\section{Results}

\subsection{Analysis on Genomic Organization.}

The Spud DB database was used to analyze the chromosomal localization and genomic organization of StPDS. The gene ID in Spud DB database is PGSC0003DMT400023666. The StPDS gene was mapped to chromosomes 3 and has 14 exons and 13 introns (Fig. 1).
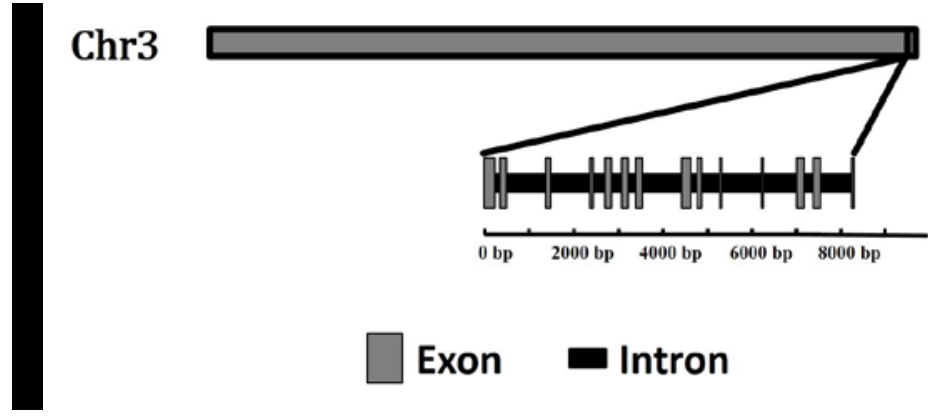

Exon

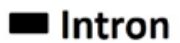

Fig. 1 Chromosomal location and genomic structure of StPDS.

\subsection{Protein Physical and Chemical Properties Analysis.}

Sequence analysis indicated that the StPDS gene contained a 1,752-bp open reading frame (ORF), which encoded a 583-amino acids protein with a calculated molecular mass of $64.97 \mathrm{kD}$ and an isoelectric point (pI) of 6.41. The amino acid types and proportions of the StPDS gene was shown in Figure 2, the highest number of amino acid is Leucine (Leu), whereas the lowest number is Tryptophan (Trp). Its predicted formula was $\mathrm{C}_{2928} \mathrm{H}_{4611} \mathrm{~N}_{771} \mathrm{O}_{847} \mathrm{~S}_{25}$. Its total average hydrophilicity index was -0.146, liposoluble index was 92.5, and instability index in solution was 48.26.

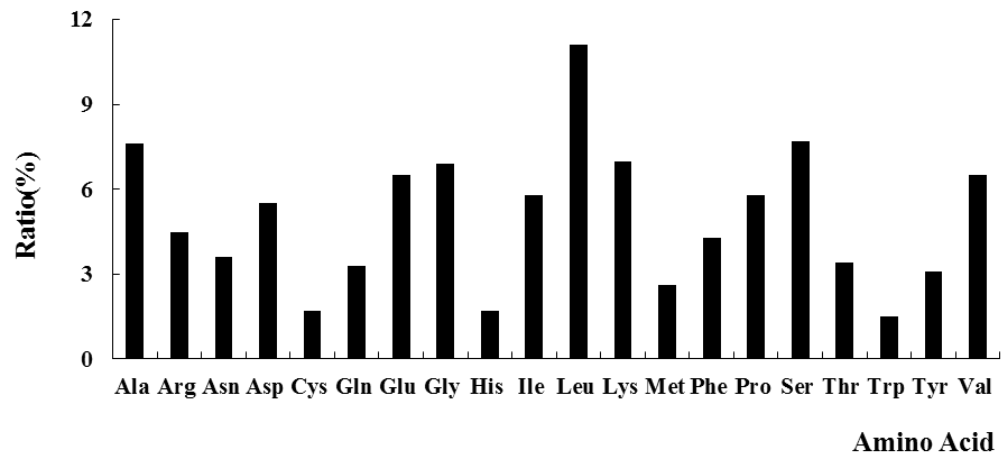

Fig. 2 Amino acid composition of StPDS 


\subsection{Subcellular Localization and Conserved Domain Analysis.}

Subcellular localization of the StPDS gene was predicted by WoLF PSORT to be in the chloroplast. The analysis using Conserved Domain Database (CDD) demonstrated that the amino acid sequence of the StPDS protein has one conserved domain PLN02612 and one COG2907 superfamily.

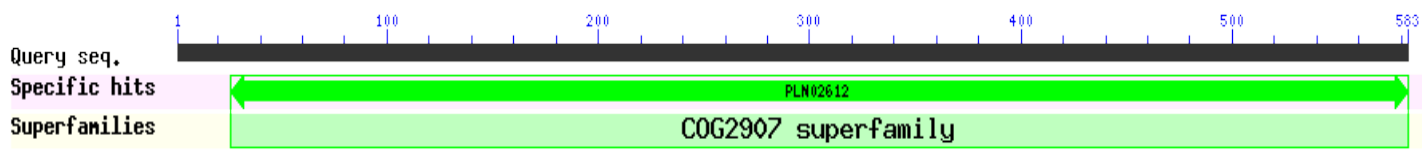

Fig. 3 Conserved domains analysis of StPDS

\subsection{Homology and Phylogenetic Tree Analysis.}

A phylogenetic tree was constructed to illustrate the relationship among the PDS proteins of potato and 18 other higher plant species (Fig. 5). A total of two major clusters were identified, one cluster includes Solanaceae, Euphorbiaceae, Asteraceae, and Leguminosae, while the other cluster includes Sterculiaceae, Euphorbiaceae, Pedaliaceae and Rosaceae. Sequence alignment indicated that the StPDS protein is more closely related to Solanum pennellii, which belonged to the Solanaceae branch.

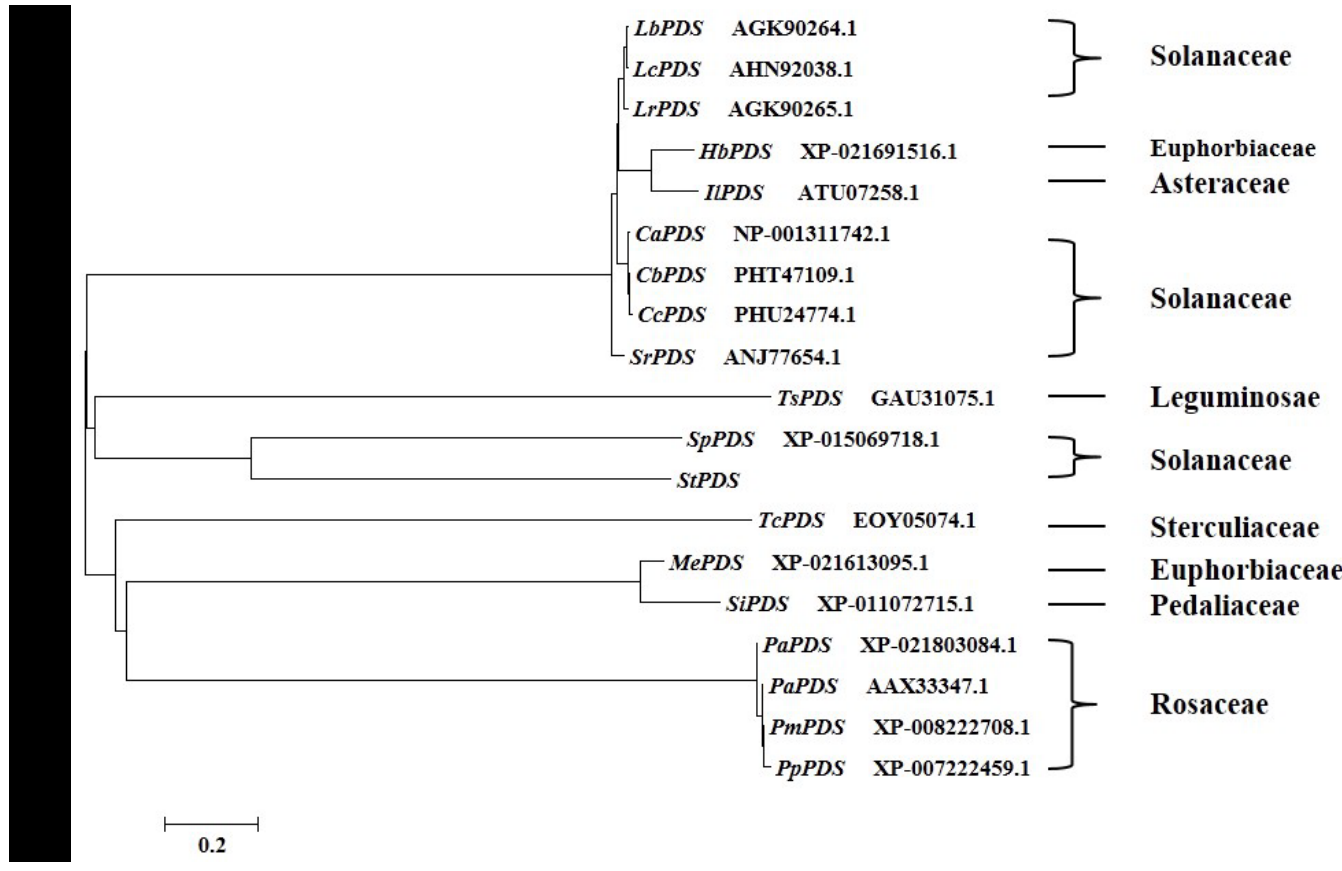

Fig. 4 Phylogenetic tree analysis of StPDS and PDS proteins of other species

\section{Discussion}

The present study analyzed the StPDS gene of potato. PDS enzyme is encoded by single-copy gene in tomato, grape, and Arabidopsis [5]. Moreover, PDS gene occurred as a single copy in potato, indicating that the enzyme may have undergone similar evolutionary patterns. Previous studies have shown that the PDS protein is relatively conserved in plants [10-11]. The PDS protein of strawberry is similar to the PDS protein of Prunus armeniaca and Carica papaya, showing 83\% and 83\% homology [10], and PDS of Strelitzia reginae exhibits 86\% homology that of Citrus $\times$ paradise [11]. The findings of the present study show that PDS from potato is highly conserved in plants, similar to that observed in earlier reports. The findings of the present study may serve as a foundation for future studies on the functions of PDS in carotenoid metabolism in potato. 


\section{Acknowledgements}

This work was supported by Science and Technology Foundation of Guizhou Province (QianKeHeJiChu[2016]1003), Talent development project of Bijie City (BiKeHe(2015)37), Science and Technology Achievement Transformation and Guidance Fund Project of Guizhou Province (QianKeHeChengZhuanZi[2014]5025), and Science and Technology Special Fund Project of Central Subsidized Place (QianKeHeTiaoZhongBuDi[2015]4003).

\section{References}

[1] M. Andersson, H. Turesson, A. Nicolia, A. Fält, M. Samuelsson, and P. Hofvander, Efficient targeted multiallelic mutagenesis in tetraploid potato (Solanum tuberosum) by transient CRISPR-Cas9 expression in protoplasts, Plant Cell Rep. 36 (2017) 117-128.

[2] S. Lu, J. Van Eck, X.J. Zhou, A.B. Lopez, D.M. O’Halloran, K.M. Cosman, B.J. Conlin, D.J. Paolillo, D.F. Garvin, J. Vrebalov, L.V. Kochian, H. Küpper, E.D. Earle, J. Cao, L. Li, the cauliflower or gene encodes a dnaJ cysteine-rich domain-containing protein that mediates high levels of beta-carotene accumulation, Plant Cell 18 (2006) 3594-3605.

[3] L. Pizarro, and C. Stange, Light-dependent regulation of carotenoid biosynthesis in plants, Cien. Inv. Agr. 36 (2009) 143-162.

[4] L.H. Liu, Z.Y. Shao, M. Zhang, and Q.M. Wang, Regulation of carotenoid metabolism in tomato, Mol. Plant 8 (2015) 28-39.

[5] N. Nisar, L. Li, S. Lu, N.C. Khin, and B.J. Pogson, Carotenoid metabolism in plants, Mol. Plant 8 (2015) 68-82.

[6] P.A. Tuan, J.K. Kim, J. Lee, W.T. Park, D.Y. Kwon, Y.B. Kim, H.H. Kim, H.R. Kim, and S.U. Park, Analysis of carotenoid accumulation and expression of carotenoid biosynthesis genes in different organs of Chinese cabbage (Brassica rapa subsp. pekinensis), Excli J. 11 (2012) 508-516.

[7] N.I. Krinsky, and E.J. Johnson, Carotenoid actions and their relation to health and disease, Mol. Aspects. Med. 26 (2005) 459-516.

[8] S.T. Mayne, Beta-carotene, carotenoids, and disease prevention in humans, FASEB J. 10 (1996) 690-701.

[9] F. Giovannucci, Tomatoes, tomato-based products, lycopene, and cancer: review of the epidemiologic literature, J. Natl. Cancer Inst. 91 (1999) 317-331.

[10]H.S. Zhu, Y.P. Li, Q.F. Wen, and H. Liu, Cloning and characterization of pds gene in Fragaria $\times$ ananassa, Acta Horticulturae Sinica 38 (2013) 55-60.

[11] M.L. Huang, and R.H. Fan, Cloning and expression analysis of phytoene desaturase in Strelitzia reginae Banks, Acta Horticulturae Sinica 40 (2013) 373-379. 